\section{Paramagnetic Resonance Centres in Amethyst and Citrine Quartz}

RECENT investigations ${ }^{1,2}$ have shown that the colour and paramagnetic resonance (PMR) centres of violet amethyst quartz are related to one another in a rather complex way. This communication reports paramagnetic resonance lines in both amethyst and citrine (which is related to amethyst by heat treatment) not analysed previously, and the behaviour of these lines with heat treatment.

In all samples of natural citrine, a broad isotropic PMR line, about $100 \mathrm{~g}$ in width, is observed (Fig. 1); its intensity is proportional to the depth of the yellow-brown colour. Both this line and the colour reach maximum intensity for annealing temperatures in the range $600^{\circ}-700^{\circ} \mathrm{C}$, and anneal out together at about $1,000^{\circ} \mathrm{C}$. When natural amethyst is annealed at about $540^{\circ} \mathrm{C}$, the violet areas turn yellow-brown; the appearance resembles that of citrine; and the broad isotropic PMR line appears. The colour of citrine has previously ${ }^{3}$ been postulated as due to a yellow ferric compound (colloidal hydrous oxide); we suggest that the PMR line is due to $\mathrm{Fe}^{3+}$ in amorphous surroundings ${ }^{4}$.

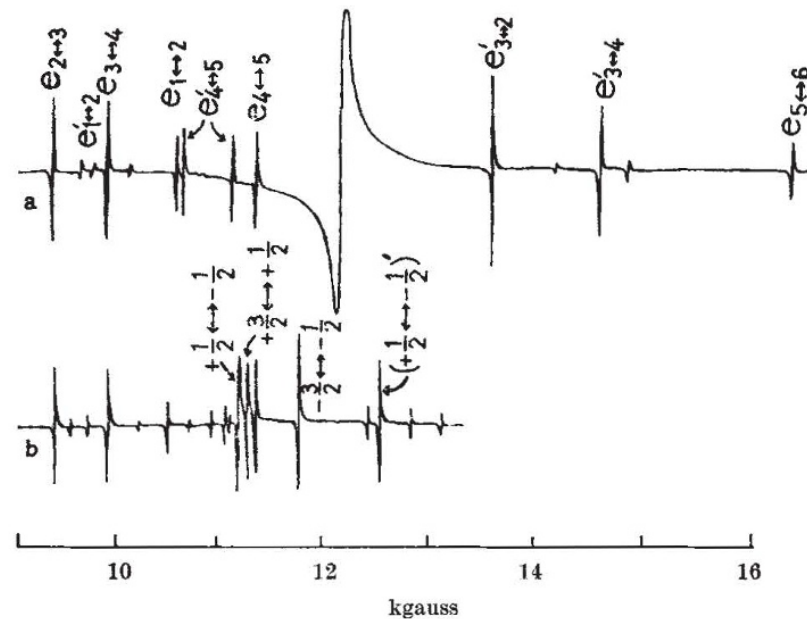
Fig. 1. $8 \mathrm{~mm}$ wave-length PMR spectra of: $(a)$ Citrine, with $B \pi a$-axis.
The lines labelled $e$ belong to the $\mathrm{Fe}^{3+}$ sites investigated by Hutton dashes denote sites with $x$-axes along the remaining $a$-axes. The energy levels have been numbered in order of increasing energy. (b) Amethyst; $B \pi x$-axis of a site with $S=3 / 2$

We have also observed that most samples of amethyst have three additional weakly populated sites for the $\mathrm{Fe}^{3+}$ centres previously analysed ${ }^{1}$. These sites have magnetic axes related to those of the strongly populated sites by a rotation of $60^{\circ}$ about the $c$-axjs. On heattreatment to temperatures greater than the $\alpha$ - to $\beta$-phase transition temperature, the line strengths for the two sets of sites become nearly equal, due presumably to diffusion either of $\mathrm{Fe}^{3+}$ ions or the alkali precursors ${ }^{1}$. Typically, for one sample of amethyst, the ratio of the line intensities of the strong sites and the weak sites fell from 400 before annealing, to 100 after annealing at $500^{\circ} \mathrm{C}$ for $15 \mathrm{~h}$, and to 1.3 after annealing at $700^{\circ} \mathrm{C}$ for $15 \mathrm{~h}$.

We have also found two further PMR centres in amethyst, for which the magnetic axes are not related in any simple way to the crystallographic axes (Fig. 2). The observed three lines associated with these centres can be fitted with a spin-Hamiltonian

$$
=g \beta B . S+1 / 3 D 0_{2}^{\circ}+E 0_{2}^{2}
$$

where the $0_{m}^{n}$ are spin operators ${ }^{5}, S=3 / 2, g=2 \cdot 004$, $D=4 \cdot 24 \mathrm{Gc} / \mathrm{s}$, and $(E / D)=0.33$ (doublet zero-field splitting, $9 \cdot 8 \mathrm{Gc} / \mathrm{s})$. The $z$-axis for a site is defined as the axis making $(E / D)$ a maximum. It was thought at first that these PMR lines might have been the stronger lines from a

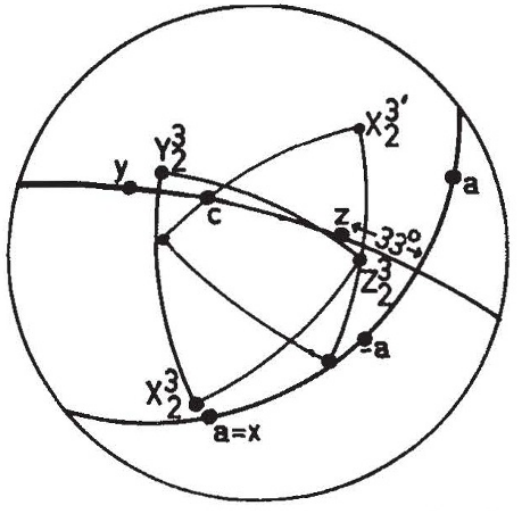
Fig. 2. Stereographic projection showing the relation of the magnetic
axes of the $\mathrm{Fe}^{3+}$ centres (ref. 1) $(x, y, z)$ and the centres with $S=3 / 2$ $\left(x_{2}^{3}\right.$, etc.) to the crystallographic axes

centre with $S=5 / 2$ (corresponding to $\mathrm{Fe}^{3+}$ ); however, a careful search did not reveal the expected lines. No certain identification for this centre, which is not characteristic of all samples of amethyst, was possible.

Further work is planned on the correlations between changes in the optical absorption bands and changes in the PMR spectra produced by heat treatment.

\section{R. HutToN}

Department of Physics,

University of Canterbury,

Christchurch, New Zealand.

\section{G. J. Troup}

Department of Physics,

Monash University,

Victoria, Australia.

${ }^{1}$ Button, D. R., Phys. Lett., 12, 310 (1964).

2 Barry, T. I., McNamara, P., and Moore, W. J., J. Chem. Phys., 42, 2599 (1965).

${ }^{3}$ Folden, E. F., Amer. Mineral., 10, 203 (1925).

- McLaren, A. C., and Phakey, P. P., Austral. J. Phys., 18, 135 (1965).

5 Orbach, R., Proc. Roy. Soc., A, 264, 458 (1961).

\section{Effect of Frequency on Texture-induced UItrasonic Wave Birefringence in Metals}

As ultrasonic shear (transverse) wave entering a stressed solid body splits into two components travelling at slightly different velocities depending on the relevant elastic 'constants' which are slightly different along the two principal stress axes normal to the wave propagation direction. Thus the velocity difference between the two orthogonally polarized shear waves propagated normal to, and polarized parallel and perpendicular to, a principal stress axis should be stress dependent because of this effect of 'acousto-elasticity' or 'sonoelasticity'. This shear wave birefringence has been used in recent years to detect internal stresses in polycrystalline metallic specimens ${ }^{1,2}$, but the interpretation of the results was difficult because the preferred grain orientation (texture) had identical effects to the internal stress. In the work described here, attempts were made with some success to separate the effects of stress and grain orientation on the ultrasonic shear wave birefringence.

Measurements have been made of the effect of ultrasonic wave frequency on the shear wave birefringence in a stainless steel sample. The stainless steel $(F V-535)$ bar selected for the purpose has a relatively large amount of preferred orientation parallel to the major axis of the bar. The ultrasonic shear wave velocity differences (birefringence) are small, but can be measured accurately by electronic techniques. The shear waves were generated by lead zirconate titanate $(P Z T-5)$ crystals mounted in a pressurecoupled transducer ${ }^{3}$ and propagated across the centre of the stainless steel bar held in a hydraulic press. 